\title{
The Veil of Obscuration: Additional Radiographic Sign of Posterior Shoulder Dislocation
}

\author{
Lana Hirai Gimber ${ }^{1}$, Mihra S. Taljanovic ${ }^{1}$, Zachary A Rockov², Elizabeth A. Krupinski ${ }^{3}$, \\ Tyson S. Chadaz ${ }^{4}$, Lee F. Rogers ${ }^{5}$
}

${ }^{1}$ Department of Medical Imaging, The University of Arizona, College of Medicine, Banner-University Medical Center, Tucson, Arizona, USA, ${ }^{2}$ The University of Arizona, College of Medicine, Tucson, Arizona, USA, ${ }^{3}$ Department of Radiology and Imaging Sciences, Emory University, Atlanta, GA, USA, ${ }^{4}$ Department of Orthopaedic Surgery, The University of Arizona, College of Medicine, Banner, University Medical Center Tucson, Tucson, Arizona, USA, ${ }^{5}$ Department of Radiology, University of Arizona Health Sciences Center, Tucson, Arizona, USA

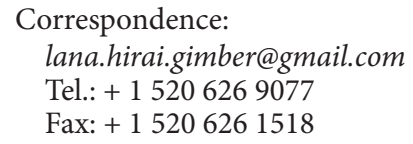

Received: 30 June 2018

Accepted: 15 August 2018

Key words: Posterior - Shoulder • Glenohumeral - Dislocation - Radiograph.
Objective. To describe a new radiographic sign, "veil of obscuration", associated with posterior glenohumeral joint (shoulder) dislocations and determine its incidence and validity compared to other known classic radiographic signs. Methods. Four-year retrospective study identified 30 acute posterior shoulder dislocation patients. Radiographs reviewed in consensus by 2 musculoskeletal radiologists for the "veil of obscuration", seen on AP shoulder radiographs and representing a comminuted fracture of the lesser tuberosity projecting over the humeral head or glenohumeral joint. Incidence of this radiographic sign of posterior glenohumeral joint dislocation in addition to other previously described classic radiographic signs, and association with other fractures, surgery, and mechanism of injury were evaluated. Continuous data was analyzed with student $t$-test and categorical data with Chi-Square test. Results. There were 20 right and 10 left posterior shoulder dislocations. Majority of injuries resulted from vehicle crash (44\%). In most cases, reverse Hill-Sachs lesion (83\%) and fixed internal rotation of the humeral head $(76 \%)$ were present, followed by trough line (43\%) and "veil of obscuration" (40\%). Trough line was seen in significantly more major trauma and vehicle crashes $(78 \%$ and $46 \% ; \mathrm{P}=0.015)$, while "veil of obscuration" was seen in more seizures $(86 \% ; \mathrm{P}=0.037)$ and in all surgical patients. No significant difference in presence of other classic radiographic signs in regards to surgery. Conclusion. The newly described radiographic sign of posterior shoulder dislocations named the "veil of obscuration" has comparable incidence as other classic radiographic signs and may be useful in the recognition and diagnosis of these injuries.

\section{Introduction}

The shoulder, or glenohumeral joint is a ball and socket construct allowing a wide range of motion. Both static and dynamic elements provide stability for this joint (1). The humeral head is significantly larger compared to the glenoid, which contributes to instability of the glenohumeral joint. Shoulder dis- locations are common, accounting for 50\% of all joint dislocations $(2,3)$, although posterior shoulder dislocations comprise less than $2-5 \%$ of all shoulder dislocations (2-4). A posterior shoulder dislocation is defined as posterior displacement of the humeral head in relation to the glenoid (Figure 1). These dislocations most frequently occur in male patients between 20 and 49 years of age 

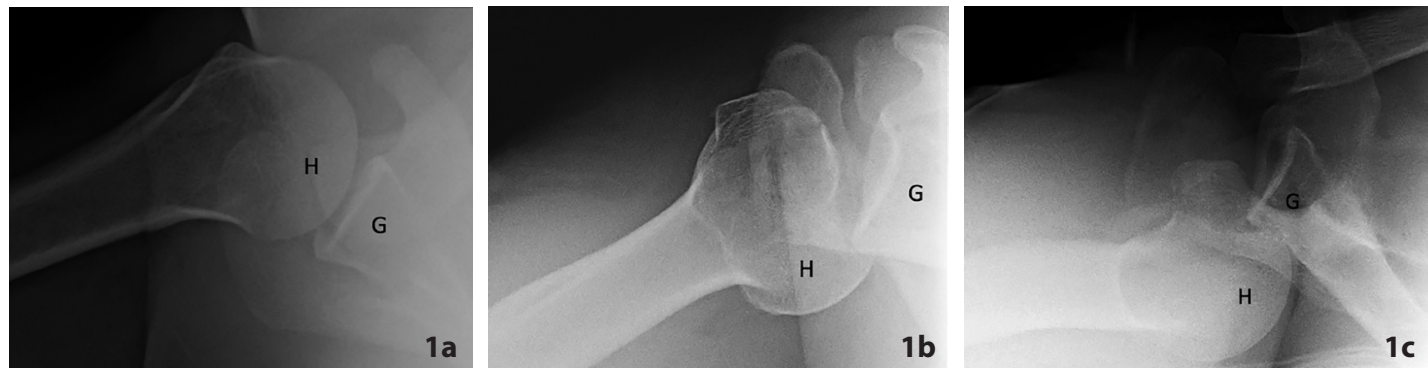

Figure 1. Axillary radiographs of the normal and posteriorly subluxed or dislocated glenohumeral joint. In (a) note normal anatomic alignment of the glenohumeral joint, with the humeral head well seated in the glenoid fossa compared to (b) which shows posterior subluxation of the glenohumeral joint, with the humeral head partially posteriorly displaced in relation to the glenoid, and (c) which demonstrates posterior dislocation of the glenohumeral joint, with the humeral head displaced completely posterior to the glenoid. $\mathrm{H}=$ humeral head, $\mathrm{G}=$ glenoid.

in addition to elderly patients over 70 years old (2). The most common mechanisms of injury include traumatic accident, seizures, and electrocution (2).

Management of posterior shoulder dislocations varies with each patient. Nonsurgical treatments include closed reduction techniques or even rarely leaving the shoulder in a chronic dislocated state $(5,6)$. Surgical treatment options include open reduction, McLaughlin or modified McLaughlin procedures for significant reverse Hill-Sachs lesions, anterior approach and bone grafting of the Hill-Sachs lesion, posterior open Bankart procedure, arthroscopic posterior Bankart repair, and arthroplasty $(5,6)$.

Although posterior shoulder dislocations are not very common, Robinson and collaborators (2011) showed that almost $18 \%$ of subjects with these injuries went on to develop recurrent instability within the first year (2). They also showed that those at highest risk for recurrent instability included age less than 40 years, large reverse Hill-Sachs lesion, and seizure as the mechanism of injury.

\section{Imaging Work-up of Posterior Shoulder Dislocations}

Routine radiologic shoulder examination usually consists of anteroposterior (AP) ra- diographs in internal and external rotation and either axillary or scapular "Y" views (5, 7) (Figure 2). While an axillary view can confirm the diagnosis, there can be limitations in obtaining this view in patients with posterior shoulder dislocations due to pain and difficulty with arm abduction (4). In these cases where it is difficult to obtain an axillary radiograph, a scapular "Y" view is recommended (8). Computed tomography (CT) may be performed to clarify or confirm the findings if clinically indicated. In addition to CT, magnetic resonance (MR) imaging and MR arthrography may help to further characterize associated bone and soft tissue injuries, but is not indicated in the acute clinical setting and may not be readily available in many academic institutions worldwide $(9,10)$.

Posterior shoulder dislocations can be overlooked on radiographs due to the subtlety of the imaging findings and difficulties in patient positioning, as well as the relative rarity of posterior shoulder dislocations (3, 7). If missed, the untreated cases can progress to chronic instability or lead to open reduction $(2,5)$. 

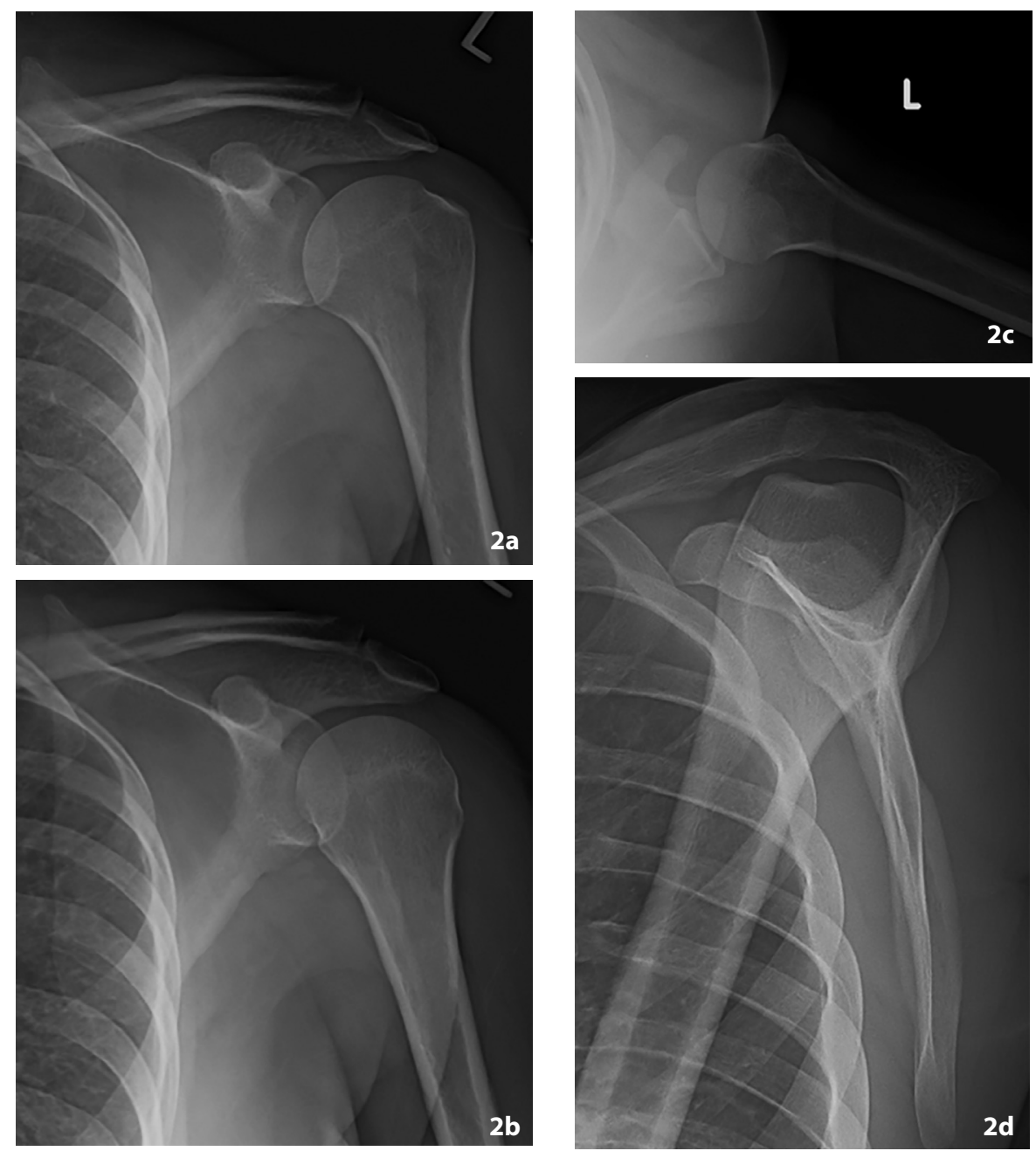

Figure 2. Routine shoulder radiographs. The complete radiographic examination of the shoulder consists of anteroposterior (AP) radiographs in (a) external rotation and (b) internal rotation and (c) an axillary view. When an axillary view cannot be obtained due to pain and difficulty with arm abduction, (d) a scapular "Y" view may be obtained.

\section{Classic Radiographic Findings of Posterior Shoulder Dislocations}

Knowledge and awareness of the radiographic findings which facilitate the diagnosis of posterior shoulder dislocations are required. These include fixed internal rotation of the humeral head (Figure 3a-b), impaction fracture at the anteromedial aspect of the humeral head (reverse Hill-Sachs lesion) (Figure 3c), widening of the glenohumeral joint space with lack of humeral head and glenoid overlap in AP projection (Fig- ure 3d), and the "trough line" (Figure 3e), a vertical line of cortical bone seen at the edge of a compression fracture at the anterior superomedial aspect of the humeral head (3, $4,11,12)$.

\section{The "Veil of Obscuration" - An Additional Radiographic Finding Associated with Posterior Shoulder Dislocations}

Imaging studies are a common part of a patient's Emergency Department visit. How- 

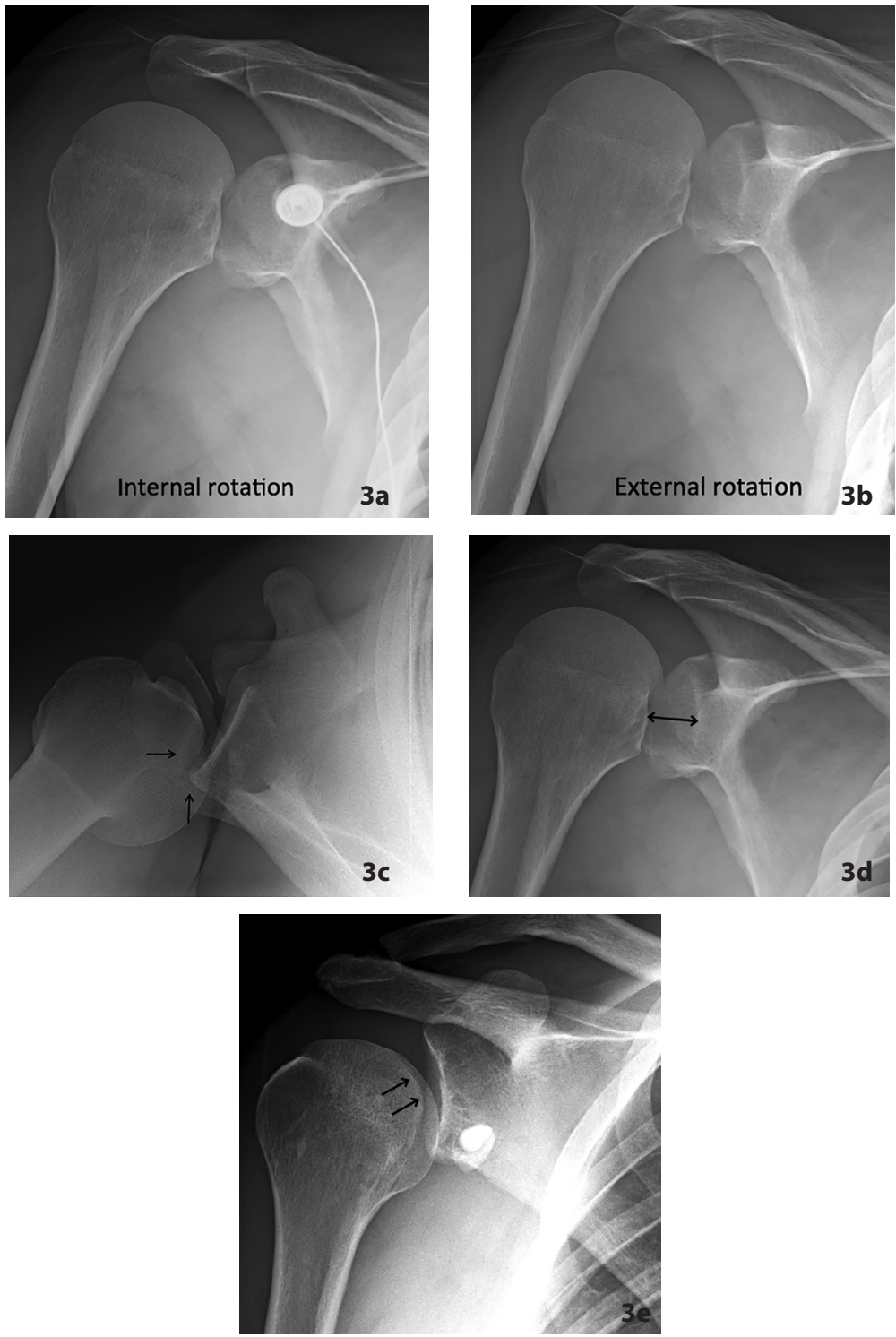

Figure 3. Classic radiographic findings of posterior shoulder dislocation. In (a) AP internal rotation and (b) attempted AP external rotation radiographs of the right shoulder, the humeral head remains in fixed internal rotation on both imaging projections. (c) Axillary radiograph of the right shoulder in a different patient shows an impaction fracture at the anteromedial aspect of the humeral head (arrows) consistent with a reverse Hill-Sachs lesion. In (d), internal rotation AP radiograph of another patient, note a widened glenohumeral joint space (arrow) measuring $1.8 \mathrm{~cm}$ with inadequate overlap between the humeral head and glenoid. (e) AP radiograph of the right shoulder in another different patient shows a vertical line of cortical bone projecting over the superomedial aspect of the humeral head (arrows) corresponding to the trough line sign created by the margin of the humeral head impaction fracture. Surgical hardware projects over the inferior glenoid from prior screw fixation.

ever, in many cases the initial imaging interpretation falls upon the emergency physician, with the final interpretation by the radiologist occurring after patient disposition and treatment plans have been made or not at all. In addition, in many academic institutions, attending radiology interpretation may not be readily available overnight. 
To further assist the emergency physician in identification of posterior shoulder dislocations, we describe an additional radiographic sign of posterior shoulder dislocation that we refer to as the "veil of obscuration" (Figures 4 and 5). This sign is seen on AP shoulder radiographs and represents a frequently comminuted fracture of the lesser tuberosity projecting over the humeral head or glenohumeral joint creating a "veil" of bone density that may obscure the underlying posterior dislocation. It is well known that the lesser tuberosity of the humeral head is susceptible to fracture in association with posterior dislocations of the glenohumeral joint. Fractures of the lesser tuberosity may be sizable, and much larger than anticipated on radiographs.

We believe that the "veil of obscuration" sign may aid in the detection of posterior
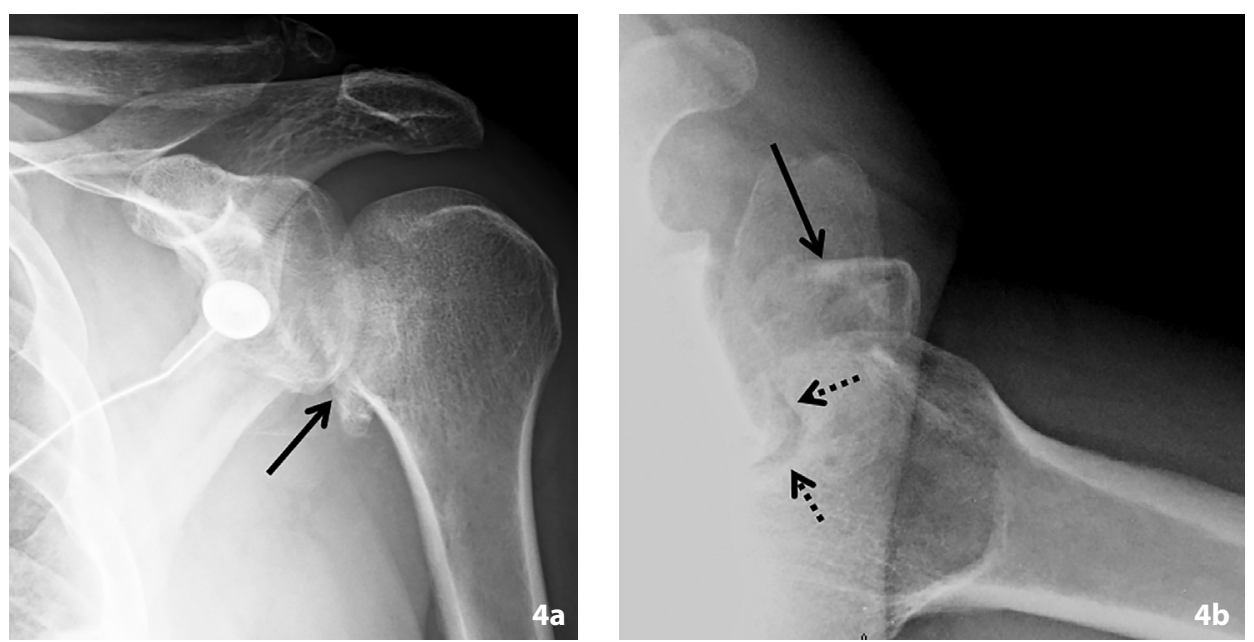

Figure 4. The "veil of obscuration" radiographic sign. (a) AP internal rotation radiograph of the left shoulder shows a displaced comminuted lesser tuberosity fracture (solid arrow) creating the "veil of obscuration" radiographic sign. (b) Axillary radiograph of the same shoulder demonstrates the displaced fracture of the lesser tuberosity (solid arrow). Also note posterior dislocation of the humeral head in relation to the glenoid with large reverse Hill-Sachs lesion at the anteromedial aspect of the humeral head (dashed arrows).
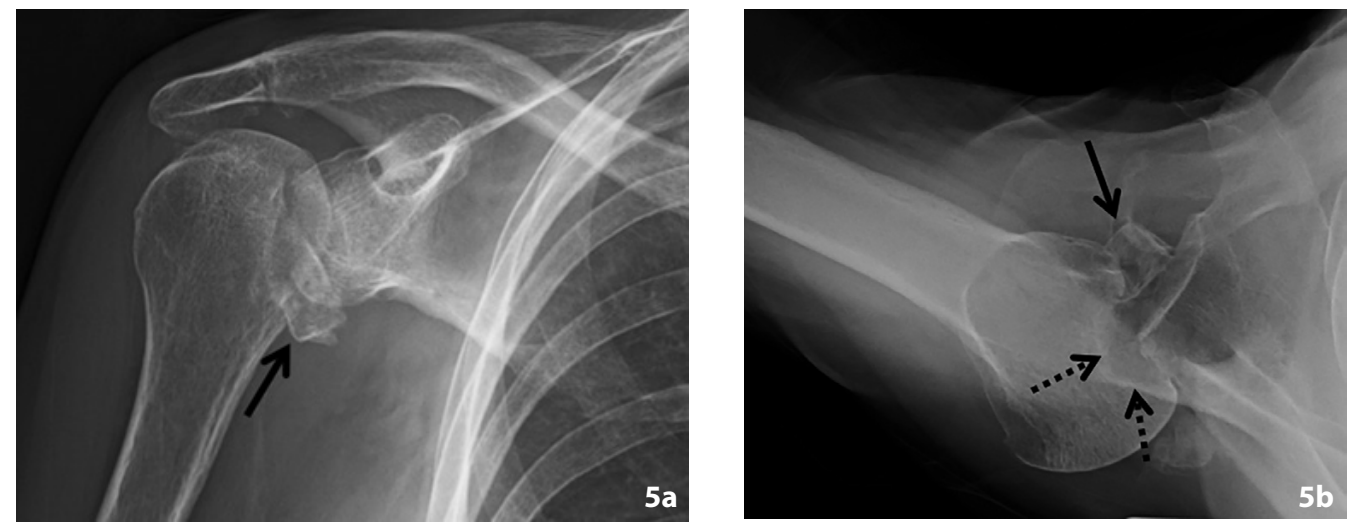

Figure 5. Sharply defined "veil of obscuration" radiographic sign. (a) AP internal rotation radiograph of the right shoulder shows a comminuted lesser tuberosity fracture (solid arrow) creating the sharply demarcated "veil of obscuration" radiographic sign. (b) Axillary radiograph of the same shoulder demonstrates a displaced fracture of the lesser tuberosity (solid arrow). Also note the humeral head is dislocated posteriorly in relation to the glenoid with large reverse Hill-Sachs lesion at the anteromedial aspect of the humeral head (dashed arrows). 

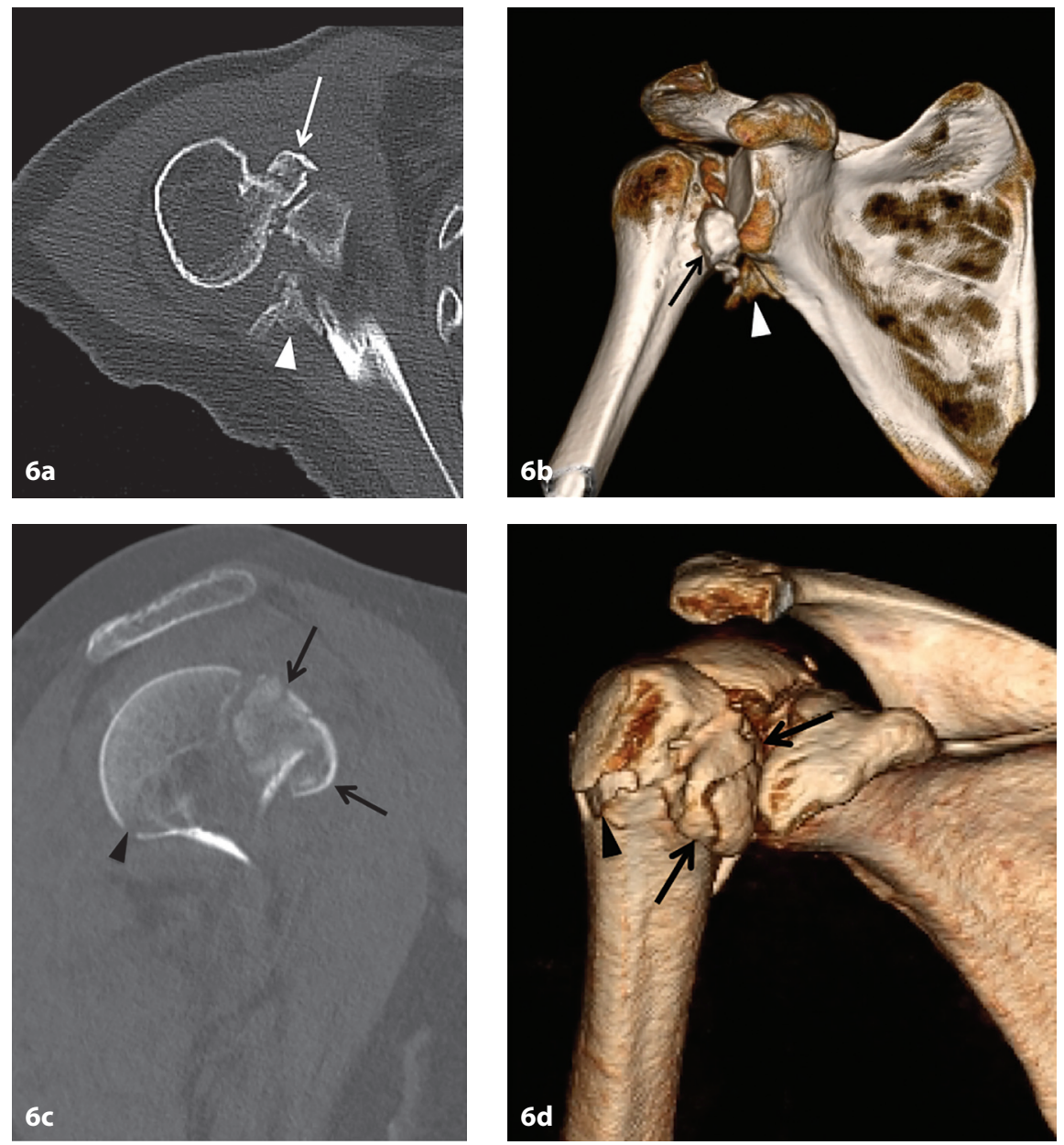

Figure 6. CT images of the "veil of obscuration" in the setting of posterior shoulder dislocations. (a) Axial and (b) 3D CT reconstruction images of the right shoulder clearly demonstrate a mildly displaced lesser tuberosity fracture fragment consistent with the "veil of obscuration" sign (arrow) and a displaced reverse bony Bankart lesion of the posterior glenoid (arrowhead). The humeral head is posteriorly subluxed. (c) Sagittal and (d) 3D CT reconstruction images of the right shoulder in another patient clearly demonstrate a mildly displaced comminuted lesser tuberosity fracture consistent with the "veil of obscuration" (arrows). Also note an associated comminuted humeral surgical neck fracture (arrowheads) as well as additional comminution of the humeral head. The humeral head is posteriorly subluxed, which was better seen on the axial CT images (not included).

glenohumeral joint dislocations. The principle fracture fragment is flat and disc-like shaped. It is typically displaced inferiorly and medially with respect to the humeral head and seen en-face on AP radiographs (Figures 4 and 5). In this projection, the fragment is usually poorly defined and appears as a veil or cloud-like density which obscures underlying bony detail (Figure 4). The "veil of obscuration" was so named because the "veil" created by the overlying displaced bone fragments often obscures the underlying posterior dislocation. Rarely, the fragment is more sharply defined on the AP projections (Figure 5). The true nature of the bone fragment is best visualized on CT images with $2 \mathrm{D}$ and $3 \mathrm{D}$ reconstruction (Figure 6) which may not be available in routine emergency department practice.

The purpose of this study was to describe and determine the incidence and validity of the "veil of obscuration" radiographic sign in posterior shoulder dislocations. 


\section{Methods}

\section{Subjects}

Institutional review board approval was obtained with waiver of consent for this retrospective study. Consecutive patient records were obtained for patients diagnosed with posterior shoulder dislocation in our institution from January 1, 2011 to January 1, 2015. Inclusion criteria were (a) patients with diagnosis of acute posterior glenohumeral joint dislocation confirmed by radiographs and (b) patients with available diagnostic imaging on our Picture Archiving and Communication System (PACS) in a major regional university hospital which demonstrates the posterior glenohumeral joint dislocation. Exclusion criteria were patients without available radiologic images or with shoulder arthroplasty.

Radiographic images of the affected shoulder were reviewed by two fellowship trained musculoskeletal radiologists with more than 30 and 3 years of experience in consensus for presence of the "veil of obscuration" representing a displaced lesser tuberosity fracture fragment and other previously described classic radiographic signs for posterior shoulder dislocation including fixed internal rotation of the humeral head, widened glenohumeral joint space, and trough sign in the AP projections, and posterior subluxation or dislocation of the humeral head in relation to the glenoid, as well as reverse Hill-Sachs lesion at the anterior superomedial aspect of the humeral head and reverse bony Bankart lesion in the axillary and/or scapular "Y" projections. The electronic medical records were also reviewed to obtain information regarding patient demographics and any correlation with a specific type of trauma or other factors. The incidence of each of the radiographic findings/ signs of posterior shoulder dislocations was calculated.

\section{Statistical Analysis}

Descriptive statistical analyses were performed. Mechanism of injury, prevalence of radiographic findings/signs, other fractures, and surgery were evaluated. Associations between the radiographic signs, other fractures, and surgery as a function of gender, age, and mechanism of injury were evaluated. Any significant differences in the radiographic signs and surgery were explored as well. Continuous data was analyzed with a student t-test and categorical data with a Chi-Square test.

\section{Results}

During the 48-month time period, there were 34 patients diagnosed with acute posterior shoulder dislocation at our busy emergency department which serves a population of at least a million people and approximately 100 or more patients with spectrum of shoulder injuries per week. Of these, 30 patients ( 26 males and 4 females) had available radiologic imaging pertaining to the diagnosis and were included in the study. All diagnoses of posterior glenohumeral joint dislocations were confirmed with radiographs, with 5 of these subjects also undergoing subsequent CT study and 1 subject undergoing subsequent MR imaging study. Females were significantly older than male patients ( $\mathrm{P}=0.0115)$; 4 females (mean age, 62.25 years; age range 53-69 years) and 26 males (mean age, 40.80 years; age range, 20-68 years). Twenty posterior dislocations were of the right shoulder and 10 of the left. The majority of posterior glenohumeral joint dislocations occurred as a result of a vehicle crash (44\%). Other mechanisms of injury included seizure, other major trauma (such as a fall), and minor trauma (such as tripping or lifting a tray). There was no significant difference $\left(\mathrm{X}^{2}=1.11, \mathrm{P}=0.775\right)$ in 
Table 1. Mechanism of Injury of Glenohumeral Joint Dislocations as a Function of Laterality

\begin{tabular}{llll}
\hline \multirow{2}{*}{ Mechanism } & Left Side & Right Side & Total \\
\cline { 2 - 4 } & $\%(n)$ & $\%(n)$ & $\%(n)$ \\
\hline Vehicle crash & $40(4)$ & $45(9)$ & $44(13)$ \\
\hline Seizure & $20(2)$ & $25(5)$ & $23(7)$ \\
\hline Other major trauma & $40(4)$ & $25(5)$ & $30(9)$ \\
\hline Minor trauma & $0(0)$ & $5(1)$ & $3(1)$ \\
\hline Total & $100(10)$ & $100(20)$ & $100(30)$ \\
\hline
\end{tabular}

mechanism of injury as a function of laterality (Table 1).

Table 2 demonstrates the prevalence of the radiographic signs of posterior shoulder dislocation. In most cases, a reverse HillSachs lesion $(83 \%)$ and fixed internal rotation of the humeral head (76\%) were present, followed by the trough line $(43 \%)$, and then the "veil of obscuration" (40\%).

Twenty-five patients with posterior glenohumeral joint dislocations had reverse Hill-Sachs fractures. In 7 cases, other fractures were also present. These included 1 of the humeral surgical neck, 1 of the greater tuberosity, 1 of the posterior rim of the glenoid (reverse bony Bankart lesion), 2 of the humeral head, and 2 of the distal clavicle or acromion.

Table 3 demonstrates significant differences in the radiographic signs as a function of mechanism of injury. The trough line was seen in significantly more cases of other
Table 2. Prevalence of Radiographic Signs of Posterior Shoulder Dislocation

\begin{tabular}{ll}
\hline Radiographic Sign & $\%(\mathrm{n})$ \\
\hline Widened joint space & $37(11)$ \\
\hline Trough line & $43(13)$ \\
\hline Veil of obscuration & $40(12)$ \\
\hline Reverse Hill-Sachs & $83(25)$ \\
\hline Other fractures & $23(7)$ \\
\hline Fixed internal rotation of humeral head & $76(22)$ \\
\hline
\end{tabular}

major trauma and vehicle crashes $(78 \%$ of all other major trauma cases and $46 \%$ of all vehicle crashes; $\mathrm{P}=0.015$ ), while the "veil of obscuration" sign was seen in significantly more cases of seizure activity ( $86 \%$ of all seizure cases; $\mathrm{P}=0.037$ ). There was no significant difference found regarding a widened glenohumeral joint space, reverse Hill-Sachs lesion, reverse bony Bankart lesion, other fractures, or fixed internal rotation of the humeral head as a function of mechanism of injury. Fixed internal rotation of the humeral head could not be evaluated in one patient due to lack of AP external rotation view on initial radiographic evaluation. The presence of the other classic radiographic signs of posterior shoulder dislocation or other fractures did not differ as a function of age or gender.

Four patients (2 males and 2 females) required surgery following attempted unsuccessful or contraindicated closed reduc-

Table 3. Radiographic Signs in Posterior Glenohumeral Joint Dislocations as a Function Mechanism of Injury

\begin{tabular}{|c|c|c|c|c|c|}
\hline \multirow{2}{*}{ Radiographic sign } & Vehicle crash & Seizure & Other major trauma & Minor Trauma & \multirow{2}{*}{$P$} \\
\hline & $\%(n)$ & $\%(n)$ & $\%(n)$ & $\%(n)$ & \\
\hline Widened joint space $(n=11)$ & $64(7)$ & $18(2)$ & $18(2)$ & $0(0)$ & NS \\
\hline Trough line $(n=13)$ & $46(6)$ & $0(0)$ & $54(7)$ & $0(0)$ & 0.015 \\
\hline Veil of obscuration $(n=12)$ & $25(3)$ & $50(6)$ & $25(3)$ & $0(0)$ & 0.037 \\
\hline Reverse Hill-Sachs $(n=25)$ & $44(11)$ & $28(7)$ & $28(7)$ & $0(0)$ & NS \\
\hline Other fractures $(n=7)$ & $29(2)$ & $29(2)$ & $29(2)$ & $14(1)$ & NS \\
\hline $\begin{array}{l}\text { Fixed internal rotation of } \\
\text { humeral head }(n=22)\end{array}$ & $36(8)$ & $27(6)$ & $32(7)$ & $5(1)$ & NS \\
\hline
\end{tabular}

$\mathrm{NS}=$ not significant. 
tion of posterior shoulder dislocation, usually related to complex injuries. There was a significant difference in gender, with $50 \%$ of females undergoing surgery versus $8 \%$ of males $(\mathrm{P}=0.021)$. There was a trend towards significance when looking at surgery and mechanism of injury, with $43 \%$ of patients with seizures leading to surgery $(\mathrm{P}=0.058)$. All surgical patients had reverse Hill-Sachs lesions and two of them presented with additional humeral head fractures.

In addition, all surgical patients had the "veil of obscuration" radiographic sign. Thirty-three percent of those with the "veil of obscuration" sign went on to surgery while no patients without this sign went on to surgery $(\mathrm{P}=0.0085)$. There was no significant difference in the presence of the other radiographic signs in regards to surgery.

\section{Discussion}

Posterior glenohumeral joint dislocations can often pose a diagnostic problem. Rowe et al. (1982) reported that $79 \%$ of posterior shoulder dislocations were missed on the clinical exam by the initial treating physician (13). Another study demonstrated that more than $50 \%$ of posterior shoulder dislocation cases can be initially missed on radiographs (7). Delays in diagnosing posterior dislocation are common, especially in older patients and those with concomitant injuries or fractures of the ipsilateral arm (7). Several reasons for delay in diagnosis have been reported which include failure of initial clinical evaluation to suspect the correct diagnosis, lack of or inadequate radiographic imaging and misinterpretation by inadequately trained physicians (7). If missed, the untreated posterior shoulder dislocations can progress to instability and/or lead to operative treatment $(2,5)$. However, in many instances these complications can be prevented by early diagnosis and prompt closed reduction.
Knowledge of radiographic signs associated with posterior shoulder dislocations are required to avoid delay in diagnosis and facilitate prompt diagnosis and timely treatment. Cisternino et al. (1977) previously described the frequency of the radiographic signs of posterior shoulder dislocation (11), not including the reverse Hill-Sachs lesion. In this study, the humeral head was held in internal rotation in $100 \%$ of posterior shoulder dislocations, followed by trough line (75\%), and a widened joint space (55\%). Similarly, when we excluded the reverse Hill-Sachs lesion, our study also demonstrated that the most frequent radiographic sign was fixed internal rotation (76\%), followed by the trough line (43\%), and then the widened joint space (37\%). Another more recent study in 2010 (12) showed the frequency of the trough line sign to be $64 \%$, followed by widened joint space (45\%), although the presence of the humeral head fixed in internal rotation was not evaluated.

Our study demonstrates that the "veil of obscuration" is an additional likely reliable radiographic sign of posterior shoulder dislocation in addition to the other classic radiographic findings. While the "veil of obscuration" is only seen in cases of a posterior glenohumeral joint dislocation with concomitant comminuted fracture of the lesser tuberosity, its prevalence is similar to that of the "trough line" (40\% versus 43\%), and is slightly more common than a widened glenohumeral joint space (37\%). The recognition of the "veil of obscuration" may be an indication for additional CT imaging for tri-dimensional evaluation of possible more complex injuries. Interestingly, the "veil of obscuration" appears to indicate a more severe case of posterior glenohumeral joint dislocation as all our patients who went on to have surgery demonstrated this radiographic sign. Additionally, in our subjects, the "veil of obscuration" is seen in significantly larger number of cases of seizures. 
In line with previously published data (2), posterior dislocations were more commonly seen in men than women in our study. Robinson et al. (2011) reported a prevalence of 1.2 per 100,000 posterior dislocations per year in men versus 0.6 per 100,000 per year in women during their study period. A portion of this discrepancy which is even more apparent in our study may be due to differences in types of activity or occupation between the male and female genders.

We also described fractures associated with posterior shoulder dislocations. Similar to a study in 2012 by Rouleau and collaborators, the reverse Hill-Sachs lesion was the most common fracture type associated with posterior shoulder dislocations (83\%), although we saw them in a greater number of cases than previously reported (29\%) (14).

In contrast to prior reported numbers, only one case of posterior shoulder dislocation was initially missed in our study. In this case, the patient had sustained a vehicle crash, with the dislocation initially overlooked by the treating clinician in the emergency department. Humerus radiographs instead of dedicated shoulder radiographs were initially obtained. Although, the posterior shoulder dislocation was missed on the initial humerus radiographs, it was seen on follow-up dedicated shoulder radiographs a few days later. The radiographic signs present were a widened glenohumeral joint space and trough line. The "veil of obscuration" was not present in this case.

\section{Conclusion}

In conclusion, we described an additional radiographic sign associated with posterior shoulder dislocation called the "veil of obscuration" which has a comparable incidence as previously described classic signs including the widened joint space or trough line. This radiographic sign is frequently seen with seizure activity and in general suggests the presence of a more severe injury. We conclude that the "veil of obscuration" sign may be an additional useful aid in the recognition and diagnosis of posterior glenohumeral joint dislocations.

\section{What is already known on this topic}

Posterior shoulder dislocations can be overlooked on radiographs due to the subtlety of imaging findings and difficulties in patient positioning, as well as the relative rarity of posterior shoulder dislocations. If missed, the untreated cases can progress to chronic instability or lead to open reduction. Knowledge and awareness of the radiographic findings which facilitate the diagnosis of posterior shoulder dislocations are required. These include fixed internal rotation of the humeral head, impaction fracture at the anteromedial aspect of the humeral head (reverse Hill-Sachs lesion), widening of the glenohumeral joint space with lack of humeral head and glenoid overlap in AP projection, and the "trough line", a vertical line of cortical bone seen at the edge of a compression fracture at the anterior superomedial aspect of the humeral head.

\section{What this study adds}

We describe an additional radiographic sign of posterior shoulder dislocation that we refer to as the "veil of obscuration" to further assist the emergency physician. This sign is seen on AP shoulder radiographs and represents a frequently comminuted fracture of the lesser tuberosity projecting over the humeral head or glenohumeral joint creating a "veil" of bone density that may obscure the underlying posterior dislocation. We described and determined the incidence and validity of the "veil of obscuration" radiographic sign in posterior shoulder dislocations. This sign has a comparable incidence as previously described classic radiographic signs, is frequently seen with seizure activity, and in general suggests the presence of a more severe injury. The "veil of obscuration" sign may be an additional useful aid in the recognition and diagnosis of posterior glenohumeral joint dislocations.

Authors' contributions: Conception and design: LG, MT, and LR; Acquisition, analysis and interpretation of data: ZR, LG, LR, and EK; Drafting the article: LG, MT, and LR; Revising the article critically for intellectual content: MT and TC; Approved final version of the manuscript: MT and LG

Conflict of interest: The authors declare that they have no conflict of interest.

\section{References}

1. Yin B, Vella J, Levine WN. Arthroscopic alphabet soup: recognition of normal, normal variants, and pathology. Orthop Clin North Am. 2010;41(3):297-308. 
2. Robinson CM, Seah M, Akhtar MA. The epidemiology, risk of recurrence, and functional outcome after an acute traumatic posterior dislocation of the shoulder. J Bone Joint Surg Am. 2011;93(17):1605-13.

3. Gor DM. The trough line sign. Radiology. 2002;224(2):485-6.

4. Cicak N. Posterior dislocation of the shoulder. J Bone Joint Surg Br. 2004;86(3):324-32.

5. Kokkalis ZT, Iliopoulos ID, Antoniou G, Antoniadou T, Mavrogenis AF, Panagiotopoulos E. Posterior shoulder fracture-dislocation: an update with treatment algorithm. Eur J Orthop Surg Traumatol. 2017;27(3):285-94.

6. Rouleau DM, Hebert-Davies J, Robinson CM. Acute traumatic posterior shoulder dislocation. J Am Acad Orthop Surg. 2014;22(3):145-52.

7. Robinson CM, Aderinto J. Posterior shoulder dislocations and fracture-dislocations. J Bone Joint Surg Am. 2005;87(3):639-50.

8. Goud A, Segal D, Hedayati P, Pan JJ, Weissman BN. Radiographic evaluation of the shoulder. European journal of radiology. 2008;68(1):2-15.
9. Expert Panel on Musculoskeletal Imaging:, Amini B, Beckmann NM, Beaman FD, Wessell DE, Bernard SA, Cassidy RC, et al. ACR Appropriateness Criteria((R)) Shoulder Pain-Traumatic. J Am Coll Radiol. 2018;15(5S):S171-88.

10. Demehri S, Hafezi-Nejad N, Fishman EK. Advanced imaging of glenohumeral instability: the role of MRI and MDCT in providing what clinicians need to know. Emerg Radiol. 2017;24(1):95103.

11. Cisternino SJ, Rogers LF, Stufflebam BC, Kruglik GD. The trough line: a radiographic sign of posterior shoulder dislocation. AJR American journal of roentgenology 1978;130(5):951-4.

12. Mouzopoulos G. The "Mouzopoulos" sign: a radiographic sign of posterior shoulder dislocation. Emerg Radiol. 2010;17(4):317-20.

13. Rowe CR, Zarins B. Chronic unreduced dislocations of the shoulder. J Bone Joint Surg Am. 1982;64(4):494-505.

14. Rouleau DM, Hebert-Davies J. Incidence of associated injury in posterior shoulder dislocation: systematic review of the literature. J Orthop Trauma. 2012;26(4):246-51. 